\title{
A new nomogram for individualized prediction of the probability of hemorrhagic transformation after intravenous thrombolysis for ischemic stroke patients
}

Yaya Wu ${ }^{1+}$, Hui Chen ${ }^{2+}$, Xueyun Liu ${ }^{1,3}$, Xiuying Cai', Yan Kong ${ }^{1}$, Hui Wang ${ }^{1}$, Yun Zhou', Juehua Zhu', Lulu Zhang ${ }^{1}$, Qi Fang ${ }^{1 *}$ and Tan $\mathrm{Li}^{1 *}$

\begin{abstract}
Background: A reliable scoring tool to detect the risk of intracerebral hemorrhage $(\mathrm{ICH})$ after intravenous thrombolysis for ischemic stroke is warranted. The present study was designed to develop and validate a new nomogram for individualized prediction of the probability of hemorrhagic transformation $(H T)$ in patients treated with intravenous (IV) recombinant tissue plasminogen activator (rt-PA).

Methods: We enrolled patients who suffered from acute ischemic stroke (AIS) with IV rt-PA treatment in our emergency green channel between August 2016 and July 2018. The main outcome was defined as any type of intracerebral hemorrhage according to the European Cooperative Acute Stroke Study II (ECASS II). All patients were randomly divided into two cohorts: the primary cohort and the validation cohort. On the basis of multivariate logistic model, the predictive nomogram was generated. The performance of the nomogram was evaluated by Harrell's concordance index (C-index) and calibration plot.

Results: A total of 194 patients with complete data were enrolled, of whom 131 comprised the primary cohort and 63 comprised the validation cohort, with $\mathrm{HT}$ rate $12.2,9.5 \%$ respectively. The score of chronic disease scale (CDS), the global burden of cerebral small vascular disease (CSVD), National Institutes of Health Stroke Scale (NIHSS) score $\geq 13$, and onset-to-treatment time $(\mathrm{OTT}) \geq 180$ were detected important determinants of $\mathrm{ICH}$ and included to construct the nomogram. The nomogram derived from the primary cohort for HT had C- Statistics of 0.9562 and the calibration plot revealed generally fit in predicting the risk of HT. Furthermore, we made a comparison between our new nomogram and several other risk-assessed scales for $\mathrm{HT}$ with receiver operating characteristic (ROC) curve analysis, and the results showed the nomogram model gave an area under curve of $0.9562(95 \% \mathrm{Cl}, 0.9221-0.9904, P<0.01)$ greater than HAT (Hemorrhage After Thrombolysis), SEDAN (blood Sugar, Early infarct and hyper Dense cerebral artery sign on noncontrast computed tomography, Age, and NIHSS) and SPAN-100 (Stroke Prognostication using Age and NIHSS) scores.

\footnotetext{
*Correspondence: fangqi_008@126.com; 504535473@qq.com

${ }^{\dagger}$ Yaya Wu and Hui Chen contributed equally to this work.

'Department of Neurology, The First Affiliated Hospital of Soochow University, Suzhou, Jiangsu Province, China

Full list of author information is available at the end of the article
}

(c) The Author(s). 2020 Open Access This article is licensed under a Creative Commons Attribution 4.0 International License, which permits use, sharing, adaptation, distribution and reproduction in any medium or format, as long as you give appropriate credit to the original author(s) and the source, provide a link to the Creative Commons licence, and indicate if changes were made. The images or other third party material in this article are included in the article's Creative Commons licence, unless indicated otherwise in a credit line to the material. If material is not included in the article's Creative Commons licence and your intended use is not permitted by statutory regulation or exceeds the permitted use, you will need to obtain permission directly from the copyright holder. To view a copy of this licence, visit http://creativecommons.org/licenses/by/4.0/ The Creative Commons Public Domain Dedication waiver (http://creativecommons.org/publicdomain/zero/1.0/) applies to the data made available in this article, unless otherwise stated in a credit line to the data. 
(Continued from previous page)

Conclusions: This proposed nomogram based on the score of CDS, the global burden of CSVD, NIHSS sCore $\geq 13$, and $\mathrm{OTT} \geq 180$ gives rise to a more accurate and more comprehensive prediction for $\mathrm{HT}$ in patients with ischemic stroke receiving IV rt-PA treatment.

Keywords: Ischemic stroke, Thrombolysis, Hemorrhagic transformation (HT), Nomogram

\section{Background}

Intravenous thrombolysis (IVT) remains the standard treatment for patients with acute ischemic stroke (AIS) within $4.5 \mathrm{~h}$ after onset [1] and can improve clinical prognosis and reduce mortality [2]. However, it may cause serious complications, especially intracerebral hemorrhage transformation (HT), which leads to deteriorations of clinical neurological function and poor prognosis, thus limiting more widespread use of IVT. Therefore, a reliable scoring tool that can identify high risk of HT is essential.

Cerebral small vessel disease (CSVD) is a common finding among patients with AIS, which accounts for about $25 \%$ of all ischemic stroke patients [3]. Magnetic resonance (MR) is the gold standard imaging of CSVD, including white hyperintensities (WMHs), perivascular spaces (PVS), cerebral microbleeds (CMBs), lacunar infarction (LI) and brain atrophy. Previous studies showed that CSVD was related to hemorrhagic transformation and clinical prognosis after IVT $[4,5]$. Recently, the concept of "total CSVD burden score", which combines WMHs, PVS, CMBs and LI to indicate the joint effect of the CSVD subtypes, has drawn more and more attention. The total CSVD burden score can capture the overall effects of CSVD better than just one or two individual features separately. It has been applied in several fields including the cognitive function, stroke types and vascular risk factors [6-8]. In addition, the previous scoring systems applied in the past few years to predict the risk of intracerebral hemorrhage do not concern CSVD [9-14]. Nomogram, a graphical statistical instrument, has been generally performed in medical decisionmaking or other specialties by calculating the continous probability of a particular outcome for an individual patient [15-17].

In the present study, we aimed to develop and validate a new nomogram including the total burden score of CSVD and other related factors to predict the probability of HT for individual stroke patients with IV rt-PA treatment who would need more intensive monitoring and the extraordinary alterness.

\section{Methods}

\section{Study population and data collection}

The present study was a retrospective analysis of prospectively collected data from the Stroke Center of the
First Hospital Affiliated of Soochow University between August 2016 and July 2018. All patients with acute cerebral infarction within $4.5 \mathrm{~h}$ from symptom onset were admitted and treated with IVT after the exclusion of the related contraindications. They underwent admission and finished MR-based imaging. We collected baseline and demographic characteristic, laboratory data and imaging information at admission and during hospitalization. The chronic disease scale (CDS) was rated from 0 to 3 , which awarded one point for each of the following: hypertension (HTN), diabetes mellitus (DM) and atrial fibrillation (AF) [12]. Four putative strong predictors of HT (the score of CDS, the global burden of CSVD, baseline National Institutes of Health Stroke Scale (NIHSS) score, and onset-to-treatment time (OTT) for IVT) constitute the present nomogram. Patients who received endovascular mechanical thrombectomy or with unavailable clinical and imaging information were excluded from the analysis.All the data obtained in this study were evaluated separately by two professional physicians and corresponding thrombolytic hemorrhage risk scores were calculated according to previously published studies [9-13].

\section{Outcome}

The HT was defined as any type of intracranial hemorrhage (ICH) according to ECASS II [18], which could be seen on non-contrast computed tomography (NCCT) of head and usually accurs within $12-36 \mathrm{~h}$ after IVT [19]. The types of HT included that: HI1 was defined as small petechiae along the margins of the infarct; $\mathrm{HI} 2$, as confluent petechiae within the infarcted area but no space-occupying effect; PH1, as blood clots in $\leq 30 \%$ of the infarcted area with some slight space-occupying effect; and PH2, as blood clots in $>30 \%$ of the infarcted area with a substantial space-occupying effect [18].

\section{The assessment of CSVD}

The structure of magnetic resonance imaging (MRI) involved in the study included diffusion-weighted imaging (DWI), 3d-TOF-MRA, FLAIR, T2-weighted, T1weighted, and gradient echo/T2\%/ susceptibility weighted sequences.

The total CSVD burden score consisted of the following four imaging markers, including: (1) WMHs: WMHs were graded according to Fazekas' scale [20]. One point 
was awarded as either confluent deep WMH (Fazekas score 2 or 3 ) or irregular periventricular WMH extending into the deep white matter (Fazekas score 3); (2) PVS: PVS were defined as small $(<3 \mathrm{~mm})$ round or linear hyperintensities on T2 images in the basal ganglia or centrum semiovale. Presence of PVS would be counted if there were moderate to severe (Semiquantitative grade 2-4) [21] PVS in the basal ganglia (1 point if present). (3) CMBs or LI: CMBs were defined as small $(<5 \mathrm{~mm})$, homogeneous, round foci of low signal intensity on gradient echo images in cerebellum, brainstem, basal ganglia, white matter, or cortico-subcortical junction [22]. Lacunes infarctions (LI) were defined as rounded or ovoid lesions, $>3$ and $<20-\mathrm{mm}$ diameter, in the basal ganglia, internal capsule, centrum semiovale, or brainstem, of CSF signal intensity on T2 and FLAIR, generally with a hyperintense rim on FLAIR, and no increased signal on DWI. One point was awarded if there is one or more lacunes or any CMB. Finally, we rated the total MRI burden of CSVD on an ordinal scale from 0 to 4 [23-25].

\section{Statistical analysis}

Included patients were randomly assigned into two cohorts (7:3): the primary cohort and the validation cohort. Models were developed from the primary cohort and evaluated in the validation cohort. Continuous variables were compared using the Mann-Whitney $U$ test for non-normally distributed variables or Student's $t$-test for normally distributed variables. Differences between proportions were assessed by Fisher's exact test or Chisquare test, where appropriate. Continuous variables were reported as the mean \pm SD or median (interquartile range), and categorical variables were described by constituent ratio. The SPSS 22.0 was used for statistical analysis of baseline data.

To identify the independent predictors of HT, a multivariable logistic regression model was performed and the variables with a probability value $<0.05$ were included in the univariate analysis. The optimal cut-off for independent parameters was determined using receiver operating characteristic (ROC) curve analysis with the highest value of Youden index, which was defined as specifity+sensitivy-1. After determining the optimal cutoff value, they were converted into dichotomous variables: lower than the cut-off value, equal to the cut-off value or higher than the cut-off value.

The nomogram including all the parameters above was constructed by $\mathrm{R}$ version 3.6.0. The nomogram was created by assigning a graphic preliminary score to each of the predictors with a point ranging from 0 to 100 , which was then summed to generate a total score, finally converted to the logit and then to an individual probability (from 0 to $100 \%$ ) of $\mathrm{ICH}$. The performance of the nomogram was evaluated by Harrell's concordance index (C-index) and the calibration plot [26]. C-index $>0.7$ generally reflects a well-fitted feature of the predictive model. The overall predictive and discriminative performance of the nomogram for predicting the probability of ICH both in primary and validation cohort were compared with HAT(Hemorrhage After Thrombolysis), SEDAN (blood Sugar, Early infarct and hyper Dense cerebral artery sign on NCCT, Age, and NIHSS), SPAN100(Stroke Prognostication using Age and NIHSS), THRIVE(Totaled Health Risks in Vascular Events) and GRASPS(Glucose, Race, Age, Sex, Systolic blood Pressure, and Severity of stroke) scores by using the estimate of the area under the receiver operating characteristic curve (AUC-ROC). The Calibration curve was performed with 1000 bootstrapped resamples for internal validation and was used to analyze the agreement between nomogram and actual observation. The packages of RMS and Hmisc were involved in this process.

\section{Results \\ Clinical features and baseline characteristics}

The flow diagram of patient inclusion and exclusion is shown in Fig. 1. A total of 194 patients were included in the study for generating the nomogram. The baseline characteristics of patients in the primary and validation cohorts are shown in the Table 1 . Of the 131 individuals comprising the primary cohort, the median age was 67 (59-74) years, and $74.0 \%$ were male, including 16 (12.2\%) HT patients. The validation cohort consisted of 39 men $(61.9 \%)$ and 24 women $(38.1 \%)$ with a median age of $70(62-78)$ years, and the overall proportion of HT was 6 (9.5\%). The difference between the two cohorts was not statistically significant (all $\mathrm{P}>0.05$ ).

\section{The optimal cut-off value and risk factors for HT after thrombolysis}

Considering the availability of variables, we determined the optimal cut-off values for predicting HT. The optimal cut-off value of NIHSS score was 13 to dichotomize patients. The optimal cut-off value of OTT for thrombolysis was $180 \mathrm{~min}$. Univariable and multivariable logistic regression analysis showed that the score of CDS, the total burden score of CSVD, NIHSS score $\geq 13$, and OTT $\geq 180$ were important determinant of HT after thrombolysis (Table 2). Sensitivity and specificity of the above variables were provided in a data supplement (Table 1).

\section{Prediction nomogram for $\mathrm{HT}$ after thrombolsis}

The significant risk factors above were used to construct the nomogram (Fig. 2). The nomogram was generated by assigning a graphic preliminary score to each of the 4 predictors with a point ranging from 0 to 100 , which 


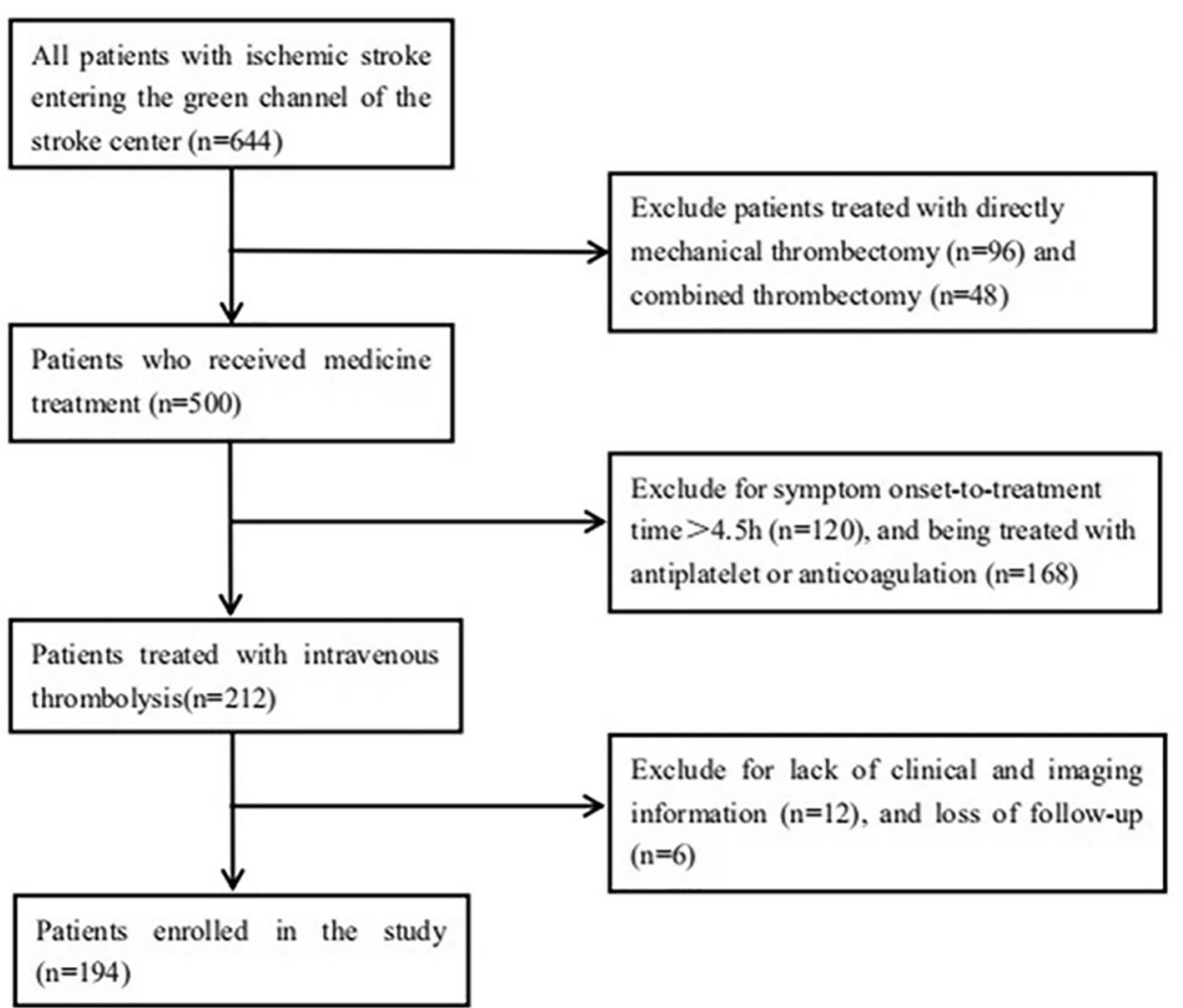

Fig. 1 Flow diagram of included and excluded patients

was then summed to generate the total score, finally converted into an individual probability of HT after thrombolysis (from 0 to 100\%). To use the nomogram, you should identify the patient's value for each predictive variable firstly, then draw a straight line upwards from each predictive value to the top point reference line and sum the points from each predictor, and finally find the location of the sum on the total points reference line and draw a straight line from total points line down to the bottom probability line to get the patient's likelihood of HT. The applicability of the nomogram can be illustrated through a clinical example (Fig. 3). The specific parameters of the nomogram model and the formula for calculating probability of $\mathrm{HT}$ are provided in the data supplement.

\section{The discrimination and calibration performance of the model}

The discriminatory ability of our model was assessed by using C-Statistics for HT prediction. The nomogram derived from the primary cohort for HT had C- Statistics of 0.9562 (95\%CI, 0.9221-0.9904), and the discriminatory ability in the validation cohort was 0.9854 (95\% CI, 0.9609-1.000).

We further compared our nomogram with HAT, SEDAN, SPAN-100 and GRASPS by using the estimate of AUC-ROC. The results showed that the new model was superior to HAT, SEDAN and SPAN-100 (all $P<$ 0.001 ) in the primary cohort, suggesting that our model provided statistically significant value for predicting HT after thrombolysis (Table 3, Fig. 4). The Table 2 (in the data supplement) showed sensitivity and specificity of nomogram and above scoring systems in the two cohorts respectively.

The calibration curves for the probability of HT after thrombolysis was demonstrated in the Fig. 5 . The $x$-axis represented the predicted HT resulted from the nomogram, and the y-axis exhibited actual HT. The calibration plot revealed general fit of the nomogram predicting the risk of HT both in the primary cohort and validation cohort.

\section{Discussion}

We presented here a new nomogram based upon the score of CDS, the total burden score of CSVD, NIHSS score, and OTT to predict the probability of HT for ischemic stroke patients treated with IV rt-PA. The discriminatory and calibration capacity of nomogram model was then confirmed by the primary cohort and the validation cohort from the same data set, and was compared with HAT, SEDAN, SPAN-100, THRIVE and GRASPS scores by AUC-ROC. 
Table 1 Baseline characteristics of the primary cohort and the validation cohort

\begin{tabular}{|c|c|c|c|c|}
\hline Baseline & $\begin{array}{l}\text { Primary cohort } \\
(n=131)\end{array}$ & $\begin{array}{l}\text { Validation cohort } \\
(n=63)\end{array}$ & $\begin{array}{r}\text { Test } \\
\text { statistic } \\
\end{array}$ & $P$ value \\
\hline Age & $67(59-74)$ & $70(62-78)$ & $-1.441^{\mathrm{a}}$ & 0.149 \\
\hline Sex, male & $97(74.0)$ & 39(61.9) & 2.992 & 0.084 \\
\hline Admission SBP mmHg & $152 \pm 21$ & $153 \pm 21$ & $-0.260^{b}$ & 0.796 \\
\hline Admission DBP mmHg & $86(78-98)$ & $85(78-92)$ & $-0.378^{a}$ & 0.705 \\
\hline Admission glucose & $5.7(4.9--6.8)$ & $6.1(5.2--7.6)$ & $-1.419^{\mathrm{a}}$ & 0.156 \\
\hline Stroke & 24(18.3) & $6(9.5)$ & 2.518 & 0.113 \\
\hline Smoke & $46(35.1)$ & 19(30.2) & 0.469 & 0.493 \\
\hline Dyslipidemia & $20(15.3)$ & $11(17.5)$ & 0.152 & 0.696 \\
\hline Antiplatelets/anticoagulation & 20(15.3) & $9(14.3)$ & 0.032 & 0.858 \\
\hline Hypertension & $92(70.2)$ & $40(63.5)$ & 0.888 & 0.346 \\
\hline Diabetes & 26(19.8) & 18(28.6) & 1.846 & 0.174 \\
\hline Atrial fibrillation & $30(22.9)$ & 21(33.3) & 2.389 & 0.122 \\
\hline CDS score & & & 4.008 & 0.253 \\
\hline 0 & $31(23.7)$ & 14(22.2) & & \\
\hline 1 & $60(45.8)$ & 22(34.9) & & \\
\hline 2 & $32(24.4)$ & 24(38.1) & & \\
\hline 3 & $8(6.1)$ & $3(4.8)$ & & \\
\hline NIHSS score & $6(3-11)$ & $6(3-12)$ & $-0.219^{a}$ & 0.827 \\
\hline OTT for thrombolysis min & $160(120-200)$ & 183(130-210) & $-1.721^{\mathrm{a}}$ & 0.085 \\
\hline Platelet ${ }^{*} 10 \wedge 9 / \mathrm{L}$ & $181(152-215)$ & 185(147-224) & $0.198^{\mathrm{a}}$ & 0.843 \\
\hline LDL mmol/L & $2.58 \pm 0.77$ & $2.51 \pm 0.62$ & $0.626^{\mathrm{b}}$ & 0.532 \\
\hline Creatinine umol/L & 70.4(59.9-80.0) & $69.9(55.4-84.0)$ & $-0.868^{\mathrm{a}}$ & 0.385 \\
\hline CRP mg/L & $3.7(1.7-13.3)$ & $4.6(1.3-10.5)$ & $-0.582^{a}$ & 0.561 \\
\hline Uric acid mmol/L & $300.2(241.0-351.3)$ & $290.4(248.8-351.3)$ & $-0.164^{a}$ & 0.870 \\
\hline CSVD score & & & 0.575 & 0.966 \\
\hline 0 & 23(17.6) & $11(17.5)$ & & \\
\hline 1 & $46(35.1)$ & 24(38.1) & & \\
\hline 2 & $39(29.8)$ & 16(25.4) & & \\
\hline 3 & $18(13.7)$ & 10(15.9) & & \\
\hline 4 & $5(3.8)$ & $2(3.2)$ & & \\
\hline HT after thrombolysis & $16(12.2)$ & $6(9.5)$ & 0.306 & 0.580 \\
\hline
\end{tabular}

Abbreviations: SBP systolic blood pressure, DBP diastolic blood pressure, NIHSS National Institutes of Health Stroke Scale, OTT onset-to-treatment time, CDS chronic disease scale, $L D L$ low-density lipoprotein, $H c y$ homocysteine, CRP C-reactive protein, CSVD cerebral small vessel disease, $H T$ hemorrhagic transformation ${ }^{a}$ represents the $Z$ value obtained by Mann-Whitney $U$ test, ${ }^{b}$ is $t$ value by Student's $t$-test, and the rest are $\lambda^{2}$ value by Chi-square test

Table 2 Univariable model and Full multivariable model for the relationship between risk factors and $\mathrm{HT}$ after thrombolysis

\begin{tabular}{llllll}
\hline & \multicolumn{2}{l}{ Univariable models } & & \multicolumn{2}{l}{ Full multivariable model } \\
\cline { 2 - 3 } \cline { 5 - 6 } \cline { 5 - 6 } & OR $(\mathbf{9 5 \% C l )}$ & $\boldsymbol{P}$ value & & OR $(\mathbf{9 5 \% C l})$ & $\boldsymbol{P}$ value \\
\hline CDS score & $6.32(2.90-16.57)$ & $<0.001$ & & $4.46(1.87-13.09)$ & 0.002 \\
CSVD score & $3.18(1.82-6.12)$ & $<0.001$ & & $2.94(1.47-6.78)$ & 0.004 \\
NIHSS $\geq 13$ & $11.04(3.53-36.49)$ & $<0.001$ & & $24.53(4.54-204.38)$ & 0.001 \\
OTT $\geq 180$ & $3.01(1.04-9.40)$ & 0.046 & & $7.77(1.50-61.93)$ & 0.026 \\
\hline
\end{tabular}

Abbreviations: CDS chronic disease scale (hypertension, diabetes mellitus, atrial fibrillation), CSVD cerebral small vascular diseases, NIHSS National Institutes of Health Stroke Scale, OTT onset-to-treatment time for thrombolysis
Nomogram is generally performed as a statistical prognostic model with the ability to generate an individual probability of a clinical event by integrating diverse prognostic and determinant variables, which meet our desire for biologically and clinically integrated models and fulfill our drive towards personalised medicine [27]. CSVD is a common finding among patients with acute ischemic stroke, and its main manifestations on magnetic resonance are white matter hyperintensities, cerebral microbleeds, peripheral vascular space, lacunar infarction and cerebral atrophy. Recent studies have 


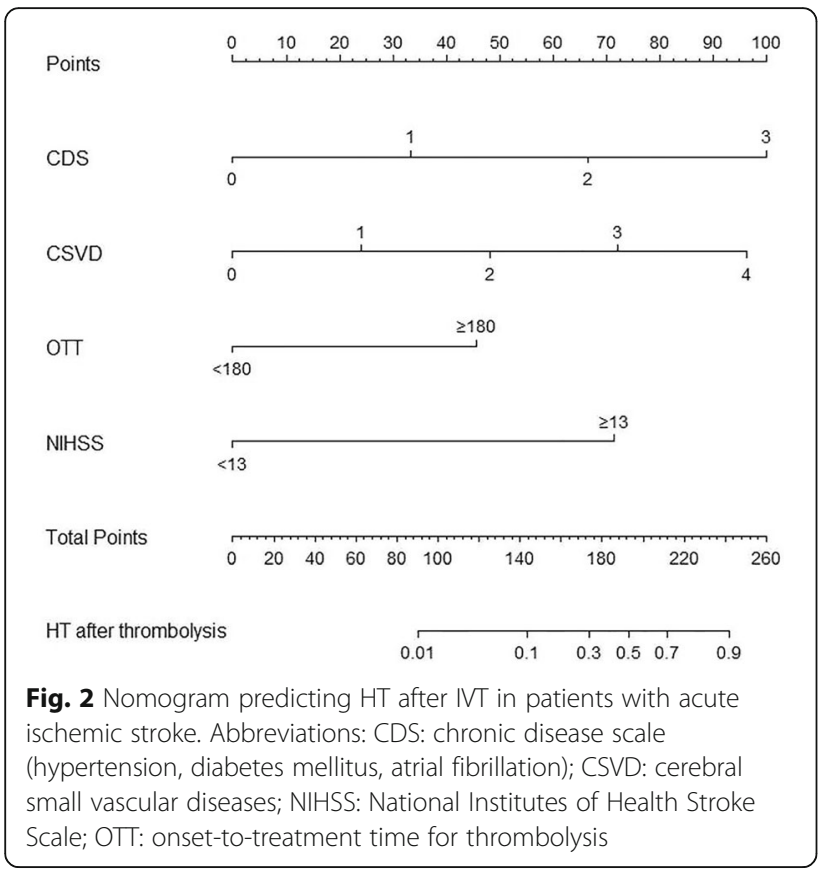

shown that white matter hyperintensities and cerebral microbleeds could increase the risk of hemorrhagic transformation after thrombolysis in stroke patients [4, 5]. Several scoring systems have been published for early prediction of ICH after thrombolysis in ischemic stroke patients [9-14]. Clearly, these scoring systems do not include any CSVD MRI markers. Besides, some recent studies have shown that the total CSVD burden score may better reflect the overall impact of CSVD on the cerebral hemisphere in a simple and practical way than considering one or two separate features [25]. Therefore, our study constructed a new nomogram model comprising the global burden of CSVD, the score of CDS, NIHS $\mathrm{S}$ score and OTT for thrombolysis. The new model presented good discriminatory ability with C- Statistics of 0.9562 (95\%CI, 0.9221-0.9904). The model has acceptable calibration suggesting a general consistency between the predicted risk and the actual risk of HT. For patients with high probability of HT, we can timely strengthen the monitoring and treatment of blood glucose and blood pressure, arrange imaging review and avoid early use of antithrombotic drugs. The model could also be used to stratify patients in large studies such as testing new devices, thrombolytic or neuroprotective drugs. Besides, if relevant software is available on a computer or mobile device, our nomogram may be easily and quickly applicable to clinicians.

The important predictor for $\mathrm{ICH}$ included in the nomogram was the total CSVD burden score $(\mathrm{OR}=2.94$, 95\%CI: 1.47-6.78, $P=0.004)$. Total CSVD burden score was a new scale proposed in recent years for more accurate evaluation of all brain injuries from CSVD.

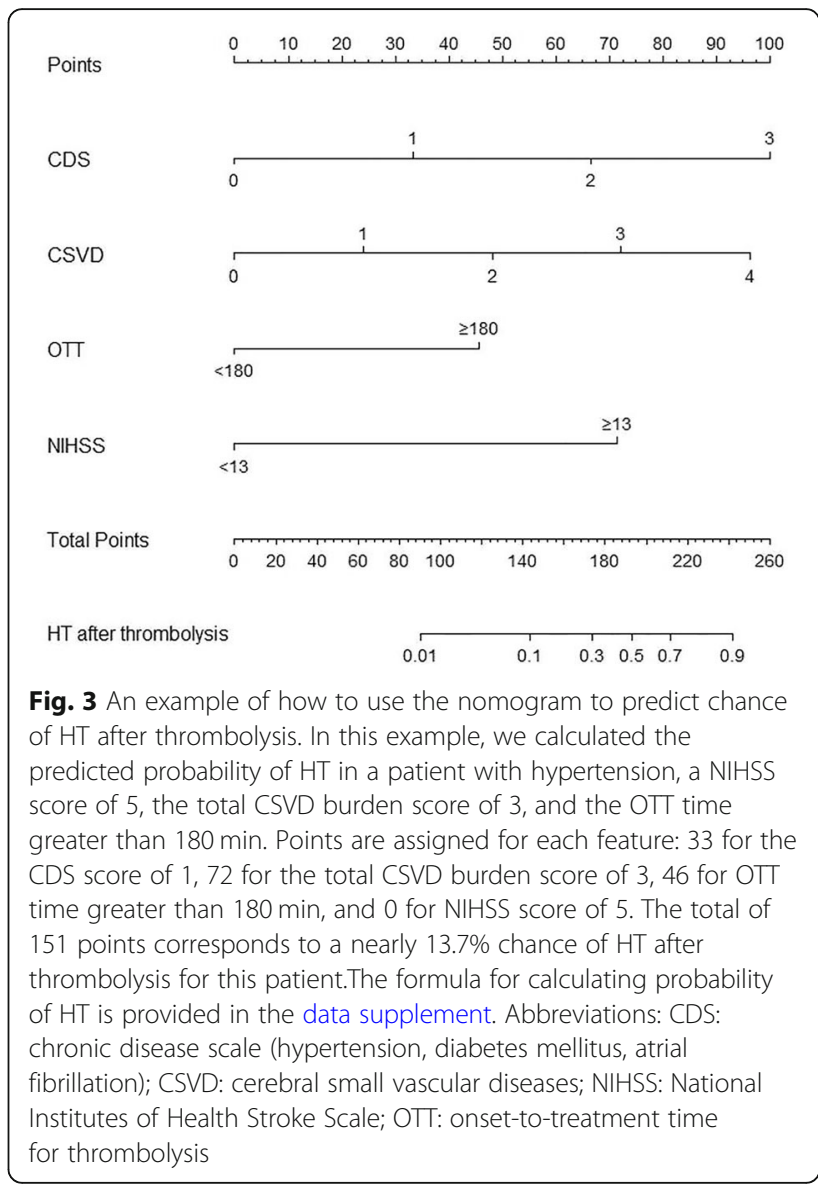

Recently, some researchers have paid attention to the relationship between total CSVD burden score and thrombolysis. Arba F et.al showed that the total CSVD burden score was correlated with disability and functional dependence [28]. Another research that combined the results of two large cohort studies pionted out that patients with a higher total CSVD burden score had a significant increased risk of recurrent ischemic stroke and hemorrhagic transformation [29]. In consistence with the above results, our single-center study found that the total CVSD burden score was a reliable predictor for poor outcomes with a suggested CSVD score $\geq$ 2 [30]. We also found that the total CSVD burden score was closely related to the transformation of bleeding after thrombolysis.

NIHSS score was another well-known predictor of HT after IV rt-PA., and it was commonly included in the existing assessment scores for hemorrhagic risk after thrombolysis. NIHSS score 7 to 12 and $\geq 13$ were the risk categories incorporated into the SITS score [14]. In SEDAN scale, the cutoff value of NIHSS was 10 point [11]. The risk categories of NIHSS score in GRASPS scale were 0 to 5,6 to 10,11 to 15,16 to 20 , and $>20$ [13]. In our study, we determined that the initial NIHSS 
Table 3 The comparison of AUC-ROC of the nomogram, HAT, SEDAN, SPAN-100, THRIVE and GRASPS for prognosis in the primary cohort and validation cohort

\begin{tabular}{|c|c|c|c|c|c|c|}
\hline \multirow[t]{2}{*}{ Scales } & \multicolumn{3}{|c|}{ Primary cohort } & \multicolumn{3}{|c|}{ Validation cohort } \\
\hline & AUC-ROC & $95 \% \mathrm{Cl}$ & $P$ value & AUC-ROC & $95 \% \mathrm{Cl}$ & $P$ value \\
\hline Nomogram & 0.9562 & $0.9221-0.9904$ & & 0.9854 & $0.9609-1.000$ & \\
\hline HAT & 0.8082 & $0.7099-0.9064$ & $<0.01$ & 0.6374 & $0.4183-0.8566$ & $<0.01$ \\
\hline SEDAN & 0.7916 & $0.6807-0.9025$ & $<0.01$ & 0.6404 & $0.4992-0.7815$ & $<0.01$ \\
\hline SPAN-100 & 0.8174 & $0.7069-0.9279$ & $<0.01$ & 0.7339 & $0.5923-0.8755$ & $<0.01$ \\
\hline THRIVE & 0.8641 & $0.7604-0.9678$ & 0.0567 & 0.7763 & $0.6423-0.9103$ & $<0.01$ \\
\hline GRASPS & 0.8239 & $0.7128-0.9350$ & 0.0148 & 0.6170 & $0.4604-0.7735$ & $<0.01$ \\
\hline
\end{tabular}

Abbreviations: HAT Hemorrhage After Thrombolysis, SEDAN blood Sugar, Early infarct and hyper Dense cerebral artery sign on NCCT, Age, and NIHSS, SPAN-100 Stroke Prognostication using Age and NIHSS, THRIVE Totaled Health Risks in Vascular Events, and GRASPS Glucose, Race, Age, Sex, Systolic blood Pressure, and Severity of stroke, AUC-ROC area under the receiver operating characteristic curve

score $\geq 13$ was independently associated with $\mathrm{ICH}$ after IV rt-PA (OR, 24.53; 95\%CI, 4.54-204.38; $P=0.001$ ), and attributed 71 scores in our model for individual prediction. The differences of the NIHSS cut-off values in the above scales might be due to the differences in study population and study methods. Therapeutic efficacy of rt-PA was time dependent and better outcomes could be expected when treatment was administered sooner rather than later within the therapeutic window. Hence, we included OTT in the model, an indicator of the quality of prehospital emergency medical service system and inhospital green channel for AIS. In consistence with Michael Mazya's reseach [14], our study proved OTT $\geq$ $180 \mathrm{~min}$ as the distinction point for HT (OR, 7.77; 95CI,
1.50-61.93; $P=0.026)$, and attributed a score of 46 points in our nomogram model. Meanwhile, our study confirmed that the score of CDS (HTN, DM, and AF) was a significant predictor for $\mathrm{HT}$ after thrombolysis (OR,4.46; 95\% CI, 1.87-13.09; $P=0.002$ ). The total score of CDS were 1, 2, and 3 that attributed a score of 33 points, 67 points, 100 points respectively.

To our knowledge, the present study was the first one of the HT assessment scales that included the total CSVD burden score. Besides, NIHSS score, the score of CDS, and OTT for thrombolysis were also included in our nomogram model to explore the risk of HT after thrombolysis. The most appealing aspect of our model was that it contained imaging biomarkers and used the
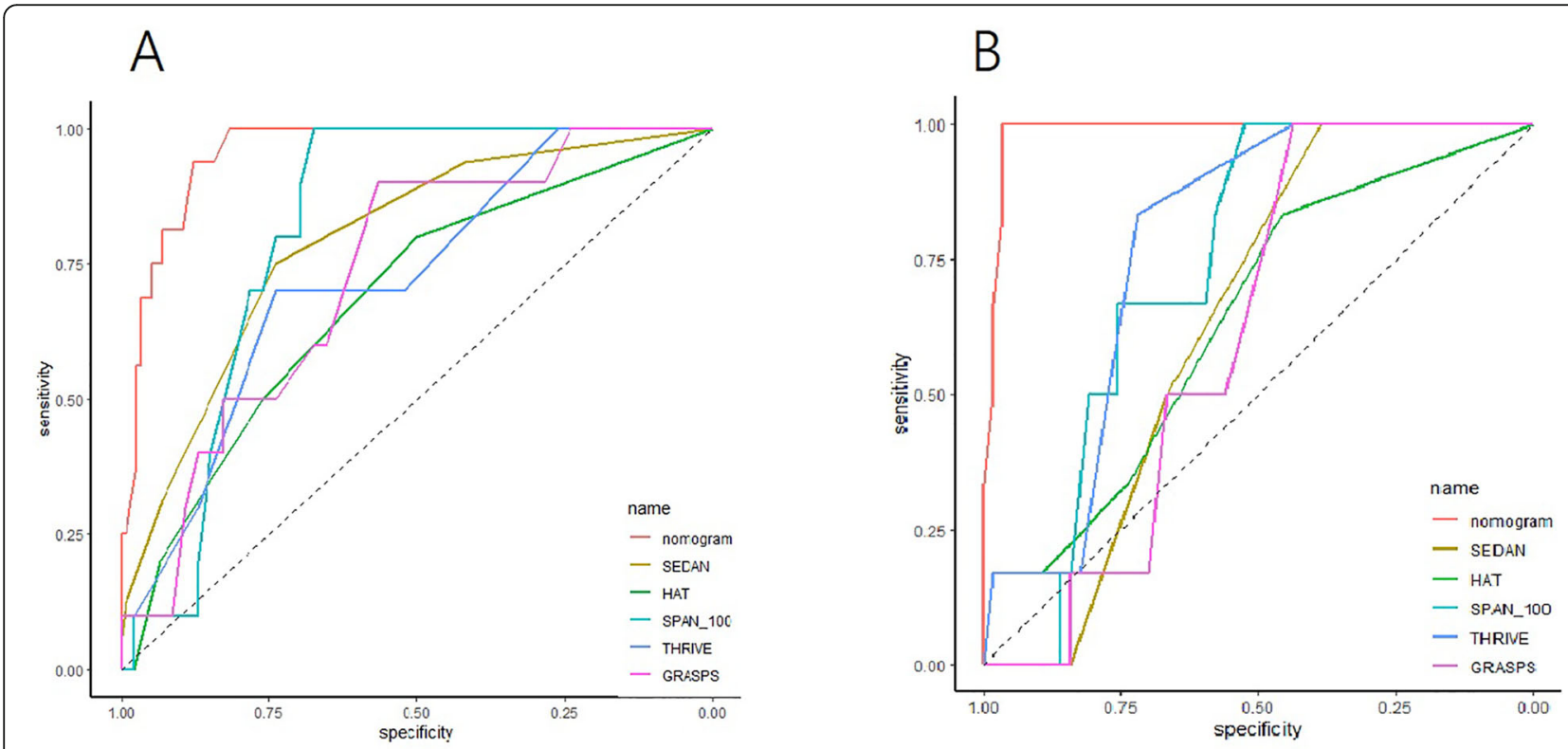

Fig. 4 Receiver operating characteristic (ROC) curve analysis for the nomogram, HAT, SEDAN, SPAN-100, THRIVE and GRASPS in the primary cohort (a) and validation cohort (b). Abbreviations: HAT(Hemorrhage After Thrombolysis), SEDAN (blood Sugar, Early infarct and hyper Dense cerebral artery sign on NCCT, Age, and NIHSS), SPAN-100(Stroke Prognostication using Age and NIHSS), THRIVE(Totaled Health Risks in Vascular Events) and GRASPS(Glucose, Race, Age, Sex, Systolic blood Pressure, and Severity of stroke) 

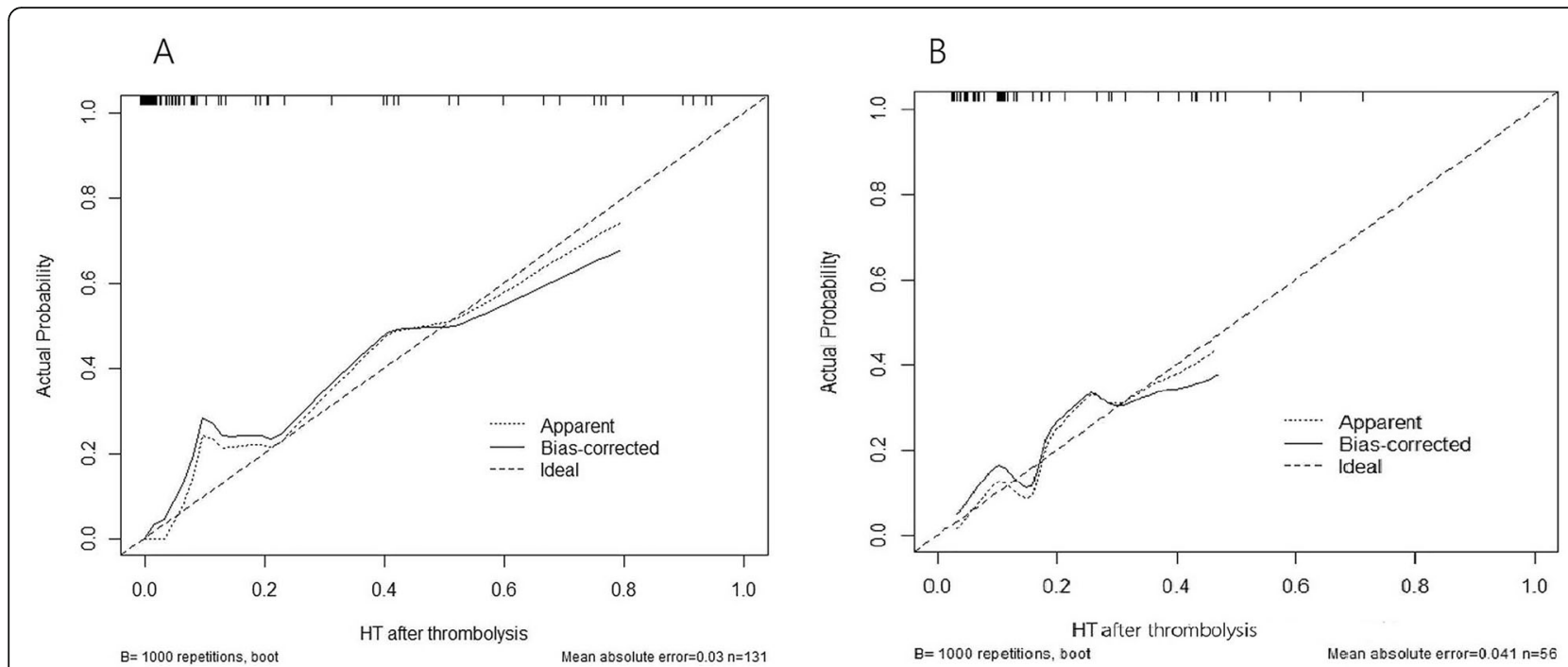

Fig. 5 The calibration curves for predicting HT of patients receiving thrombolysis in the primary cohort (a) and validation cohort (b). Abbreviations: HAT(Hemorrhage After Thrombolysis), SEDAN (blood Sugar, Early infarct and hyper Dense cerebral artery sign on NCCT, Age, and NIHSS), SPAN-100(Stroke Prognostication using Age and NIHSS), THRIVE(Totaled Health Risks in Vascular Events) and GRASPS(Glucose, Race, Age, Sex, Systolic blood Pressure, and Severity of stroke)

global load score of CSVD to reflect overall brain damage. Moreover, we used discrimination and calibration to assess the performance of our model, and the results were satisfactory.

There are still some limitations in the present study. Firstly, it is a single-center retrospective analysis with a small sample. Although the discriminative performance of the model was good, an external validation in a completely different cohort was still warranted before the formal routine clinical practice. Besides, data on platelet count, international normalized ratio, and activated partial thromboplastin time values are not available in the model to assess their possible association with HT. Lastly, a few patients with severe symptomatic intracranial hemorrhage and subarachnoid hemorrhage who could not bear MR imaging were not included in our study, so this might lead to bias in case selection.

\section{Conclusions}

We proposed and validated a nomogram model that included the score of CDS, the total burden of CSVD, NIHSS score and OTT for thrombolysis, which reliably calculated the probability of HT in AIS patients receiving intravenous rt-PA.

\section{Supplementary Information}

Supplementary information accompanies this paper at https://doi.org/10. 1186/s12883-020-02002-w.

Additional file 1: Table S1. Sensitivity and specificity of the variables concluded in nomogram. Table S2. Sensitivity and specificity of nomogram, HAT, SEDAN, SPAN-100, THRIVE and GRASPS in the primary cohort and validation cohort.

\section{Abbreviations}

AF: Atrial fibrillation; AIS: Acute ischemic stroke; AUC-ROC: Area under the receiver operating characteristic curve; CDS: Chronic disease scale; CMBs: Cerebral microbleeds; CSVD: Cerebral small vascular disease; DM: Diabetes mellitus; DWI: Diffusion-weighted imaging; ECASS II: European Cooperative Acute Stroke Study II; GRASPS: Glucose, Race, Age, Sex, Systolic blood Pressure, and Severity of stroke; HAT: Hemorrhage After Thrombolysis; HT: Hemorrhagic transformation; HTN: Hypertension; ICH: Intracerebral hemorrhage; IV: Intravenous; IVT: Intravenous thrombolysis; LI: Lacunar infarction; MRI: Magnetic resonance imaging; NCCT: Non-contrast computed tomography; NIHSS: National Institutes of Health Stroke Scale; OTT: Onset-totreatment time; PVS: Perivascular spaces; rt-PA: Recombinant tissue plasminogen activator; SD: Standard deviation; SEDAN: Blood Sugar, Early infarct and hyper Dense cerebral artery sign on non-contrast computed tomography, Age, and NIHSS; SPAN-100: Stroke Prognostication using Age and NIHSS; THRIVE: Totaled Health Risks in Vascular Events; WMHs: White hyperintensities

\section{Acknowledgments}

We thank the Department of Neurology of The First Affiliated Hospital of Soochow University (Suzhou, China) for their helpful and continuous support.

\section{Authors' contributions}

QF, TL and XC designed this study. YW and HC collected and analyzed the patient data, and were the major contributor in writing the manuscript. $X L$, $Y K, H W, Y Z, J Z$, and LZ conducted the clinical assessments and follow-up of participants. The author(s) read and approved the final manuscript.

\section{Funding}

The present study was funded by National Key R\&D Program of China (2017YFE0103700) and The social development project of jiangsu(BE2016670).

\section{Availability of data and materials}

The datasets generated during the current study are available from the corresponding author on reasonable request. 


\section{Ethics approval and consent to participate}

The use of data analysis was legally approved by the ethics committees of the First Hospital Affiliated to Soochow University (No.2020157) without need for consent.This research was performed in accordance with the tenets of the Declaration of Helsinki as amended in 2008.

\section{Consent for publication}

Not applicable.

\section{Competing interests}

The authors declare that they have no conflict of interest.

\section{Author details}

'Department of Neurology, The First Affiliated Hospital of Soochow University, Suzhou, Jiangsu Province, China. ${ }^{2}$ Department of Intensive Care Medicine, The First Affiliated Hospital of Soochow University, Suzhou, Jiangsu Province, China. ${ }^{3}$ Department of Neurology, The Second Hospital of Anhui Medical University, Hefei, Anhui Province, China.

\section{Received: 5 May 2020 Accepted: 16 November 2020}

\section{Published online: 24 November 2020}

\section{References}

1. Powers WJ, Rabinstein AA, Ackerson T, Adeoye OM, Bambakidis NC, Becker $K$, et al. Guidelines for the early management of patients with acute ischemic stroke: 2019 update to the 2018 guidelines for the early management of acute ischemic stroke: a guideline for healthcare professionals from the American Heart Association/American Stroke Association. Stroke. 2019;50(12):e344-418.

2. Wardlaw JM, Murray V, Berge E, del Zoppo GJ. Thrombolysis for acute ischaemic stroke. Cochrane Database Syst Rev. 2014;(7):CD000213.

3. Wardlaw JM, Smith C, Dichgans M. Mechanisms of sporadic cerebral small vessel disease: insights from neuroimaging. Lancet Neurol. 2013; 12(5):483-97.

4. Charidimou A, Pasi M, Fiorelli M, Shams S, von Kummer R, Pantoni L, et al. Leukoaraiosis, cerebral hemorrhage, and outcome after intravenous thrombolysis for acute ischemic stroke: a meta-analysis (v1). Stroke. 2016; 47(9):2364-72

5. Zand R, Tsivgoulis G, Singh M, McCormack M, Goyal N, Ishfaq MF, et al. Cerebral microbleeds and risk of intracerebral hemorrhage post intravenous thrombolysis. J Stroke Cerebrovasc Dis. 2017;26(3):538-44.

6. Staals J, Booth T, Morris Z, Bastin ME, Gow AJ, Corley J, et al. Total MRI load of cerebral small vessel disease and cognitive ability in older people. Neurobiol Aging. 2015;36(10):2806-11.

7. Pico F, Labreuche J, Touboul PJ, Leys D, Amarenco P. Intracranial arterial dolichoectasia and small-vessel disease in stroke patients. Ann Neurol. 2005; 57(4):472-9

8. Brenner D, Labreuche J, Pico F, Scheltens P, Poirier O, Cambien F, et al. The renin-angiotensin-aldosterone system in cerebral small vessel disease. J Neurol. 2008;255(7):993-1000.

9. Lou M, Safdar A, Mehdiratta M, Kumar S, Schlaug G, Caplan L, et al. The HAT score: a simple grading scale for predicting hemorrhage after thrombolysis. Neurology. 2008;71(18):1417-23.

10. Saposnik G, Guzik AK, Reeves M, Ovbiagele B, Johnston SC. Stroke prognostication using age and NIH stroke scale: SPAN-100. Neurology. 2013; 80(1):21-8.

11. Strbian D, Engelter S, Michel P, Meretoja A, Sekoranja L, Ahlhelm FJ, et al. Symptomatic intracranial hemorrhage after stroke thrombolysis: the SEDAN score. Ann Neurol. 2012:71(5):634-41.

12. Flint AC, Faigeles BS, Cullen SP, Kamel H, Rao VA, Gupta R, et al. THRIVE score predicts ischemic stroke outcomes and thrombolytic hemorrhage risk in VISTA. Stroke. 2013;44(12):3365-9.

13. Menon BK, Saver JL, Prabhakaran S, Reeves M, Liang L, Olson DM, et al. Risk score for intracranial hemorrhage in patients with acute ischemic stroke treated with intravenous tissue-type plasminogen activator. Stroke. 2012 43(9):2293-9.

14. Mazya M, Egido JA, Ford GA, Lees KR, Mikulik R, Toni D, et al. Predicting the risk of symptomatic intracerebral hemorrhage in ischemic stroke treated with intravenous alteplase: safe implementation of treatments in stroke (SITS) symptomatic intracerebral hemorrhage risk score. Stroke. 2012:43(6): 1524-31.
15. Jehi L, Yardi R, Chagin K, Tassi L, Russo GL, Worrell G, et al. Development and validation of nomograms to provide individualised predictions of seizure outcomes after epilepsy surgery: a retrospective analysis. Lancet Neurol. 2015;14(3):283-90.

16. Cappellari M, Turcato G, Forlivesi S, Zivelonghi C, Bovi P, Bonetti B, et al. STARTING-SICH nomogram to predict symptomatic intracerebral hemorrhage after intravenous thrombolysis for stroke. Stroke. 2018;49(2): 397-404.

17. Cappellari M, Mangiafico S, Saia V, Pracucci G, Nappini S, Nencini P, et al. IER-SICH nomogram to predict symptomatic intracerebral hemorrhage after thrombectomy for stroke. Stroke. 2019:50(4):909-16.

18. Larrue V, von Kummer RR, Muller A, Bluhmki E. Risk factors for severe hemorrhagic transformation in ischemic stroke patients treated with recombinant tissue plasminogen activator: a secondary analysis of the European-Australasian Acute Stroke Study (ECASS II). Stroke. 2001;32(2): 438-41.

19. Nguyen-Huynh MN, Klingman JG, Avins AL, Rao VA, Eaton A, Bhopale S, et al. Novel telestroke program improves thrombolysis for acute stroke across 21 hospitals of an integrated healthcare system. Stroke. 2018; 49(1):133-9.

20. Fazekas F, Chawluk JB, Alavi A, Hurtig HI, Zimmerman RA. MR signal abnormalities at $1.5 \mathrm{~T}$ in Alzheimer's dementia and normal aging. AJR Am J Roentgenol. 1987;149(2):351-6

21. Doubal FN, MacLullich AM, Ferguson KJ, Dennis MS, Wardlaw JM. Enlarged perivascular spaces on MRI are a feature of cerebral small vessel disease. Stroke. 2010:41(3):450-4.

22. Wardlaw JM, Smith EE, Biessels GJ, Cordonnier C, Fazekas F, Frayne R, et al. Neuroimaging standards for research into small vessel disease and its contribution to ageing and neurodegeneration. Lancet Neurol. 2013;12(8): 822-38.

23. Huijts M, Duits A, van Oostenbrugge RJ, Kroon AA, de Leeuw PW, Staals J. Accumulation of MRI markers of cerebral small vessel disease is associated with decreased cognitive function. A study in first-ever lacunar stroke and hypertensive patients. Front Aging Neurosci. 2013;5:72

24. Staals J, Makin SD, Doubal FN, Dennis MS, Wardlaw JM. Stroke subtype, vascular risk factors, and total MRI brain small-vessel disease burden. Neurology. 2014:83(14):1228-34.

25. Klarenbeek P, van Oostenbrugge RJ, Rouhl RP, Knottnerus IL, Staals J. Ambulatory blood pressure in patients with lacunar stroke: association with total MRI burden of cerebral small vessel disease. Stroke. 2013;44(11):2995-9.

26. Alba AC, Agoritsas T, Walsh M, Hanna S, lorio A, Devereaux PJ, et al. Discrimination and calibration of clinical prediction models: users' guides to the medical literature. JAMA. 2017;318(14):1377-84

27. Balachandran VP, Gonen M, Smith JJ, DeMatteo RP. Nomograms in oncology: more than meets the eye. Lancet Oncol. 2015;16(4):e173-80.

28. Arba F, Inzitari D, Ali M, Warach SJ, Luby M, Lees KR, et al. Small vesse disease and clinical outcomes after IV rt-PA treatment. Acta Neurol Scand. 2017:136(1):72-7.

29. Lau KK, Li L, Schulz U, Simoni M, Chan KH, Ho SL, et al. Total small vessel disease score and risk of recurrent stroke: validation in 2 large cohorts. Neurology. 2017;88(24):2260-7.

30. Liu X, Li T, Diao S, Cai X, Kong Y, Zhang L, et al. The global burden of cerebral small vessel disease related to neurological deficit severity and clinical outcomes of acute ischemic stroke after IV rt-PA treatment. Neurol Sci. 2019:40(6):1157-66.

\section{Publisher's Note}

Springer Nature remains neutral with regard to jurisdictional claims in published maps and institutional affiliations. 